\title{
Atomistic-Scale Simulations of Defect Formation in Graphene Under Noble Gas Ion Irradiation
}

\author{
Kichul Yoon ${ }^{\mathrm{a}}$, Ali Rahnamoun ${ }^{\mathrm{a}}$, Jacob L. Swett ${ }^{\mathrm{b}}$, Vighter Iberi ${ }^{\mathrm{c}, \mathrm{d}}{ }^{\text {, David A. Cullen }}{ }^{\mathrm{e}}$, Ivan V. \\ Vlassiouk $^{\mathrm{f}}$, Alex Belianinov ${ }^{\mathrm{d}, \mathrm{g}}$, Xiahan Sang ${ }^{\mathrm{d}}$, Olga S. Ovchinnikova ${ }^{\mathrm{d}, \mathrm{g}}$, Adam J. Rondinone ${ }^{\mathrm{d}}$, \\ Raymond R. Unocic ${ }^{\mathrm{d}}$, and Adri C.T. van Duin ${ }^{\mathrm{a}, *}$ \\ a Department of Mechanical and Nuclear Engineering, The Pennsylvania State University, \\ University Park, PA 16802, USA \\ ${ }^{\mathrm{b}}$ Advanced Technology Center, Lockheed Martin Space Systems Company, Palo Alto, CA \\ 94304, USA \\ ${ }^{c}$ Department of Materials Science and Engineering, University of Tennessee, Knoxville, TN \\ 37996, USA \\ ${ }^{\mathrm{d}}$ Center for Nanophase Materials Sciences, ${ }^{\mathrm{e}}$ Materials Science and Technology Division, ${ }^{\mathrm{f}}$ \\ Energy \& Transportation Science Division, ${ }^{\mathrm{g}}$ Institute for Functional Imaging of Materials, Oak \\ Ridge National Laboratory, Oak Ridge, TN 37831, USA
}

*Corresponding author.

Tel: +1 814863 6277. E-mail address: acv13@psu.edu (Adri C.T. van Duin) 


\section{S1. Force field parameterization results}

In order to develop the ReaxFF force field for the description of short-range nuclear repulsion, we obtained energies in the geometry that consists of a noble gas ion and benzene by

using DFT and ZBL potential ${ }^{1}$. Note that the benzene molecule, which is similar to graphene in that it is also a planar structure, was used for the interaction of ions with graphene. DFT calculation was performed with $6-311 \mathrm{G}^{* *}$ (for the interaction of benzene with $\mathrm{He}, \mathrm{Ne}$, Ar ions) and LACV3P** basis set (for the interaction of benzene with $\mathrm{Kr}$ ion) and B3LYP functional. Three impact positions (center of ring, center of bond, and top of $\mathrm{C}$ atom) were considered for the short-range repulsive interactions between ions and graphene, as indicated in the subset images in Fig. S1. The relative energies obtained from DFT, ZBL potential, and the ReaxFF force field are in good agreement with each other. 

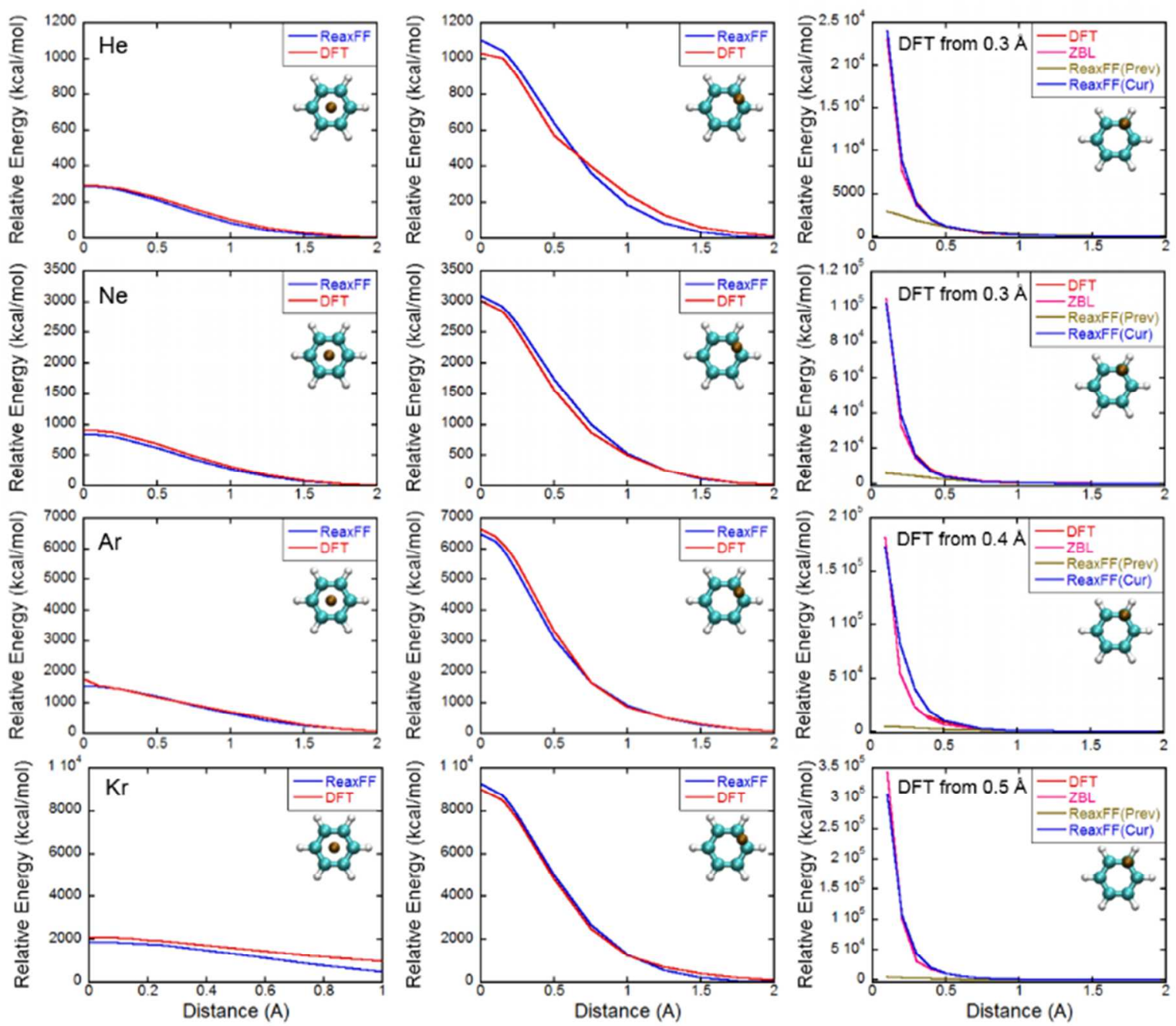

Figure S1. Force field parameterization results: Change in energy with respect to the distance between ion and benzene plane was obtained by DFT and ZBL potential, and the ReaxFF force field was parameterized against the DFT and ZBL data. Energy of the geometry, in which the distance between ion and benzene plane was shorter than $0.3 \AA$ for $\mathrm{He}$ and $\mathrm{Ne}, 0.4 \AA$ for $\mathrm{Ar}$, and $0.5 \AA$ for $\mathrm{Kr}$, were not able to be obtained in DFT. The Relative energies obtained by DFT and ZBL potential are so similar that the two energy curves are overlapped. 


\section{S2. Force field parameters for noble gas ion irradiations}

\begin{tabular}{|c|c|c|c|c|c|c|c|c|}
\hline \multicolumn{9}{|c|}{ Reactive MD-force field: C-2013 + He/Ne/Ar/Kr } \\
\hline 39 & $\begin{aligned} ! \\
\end{aligned}$ & \multicolumn{7}{|c|}{ Number of general parameters } \\
\hline & 50.0000 & \multicolumn{7}{|c|}{ !Overcoordination parameter } \\
\hline & 9.5469 & \multicolumn{7}{|c|}{ !Overcoordination parameter } \\
\hline & 1.6725 & \multicolumn{7}{|c|}{ !Valency angle conjugation parameter } \\
\hline & 1.7224 & \multicolumn{7}{|c|}{ !Triple bond stabilisation parameter } \\
\hline & 6.8702 & \multicolumn{7}{|c|}{ Triple bond stabilisation parameter } \\
\hline & 50.4850 & \multicolumn{7}{|c|}{ !c2-correction } \\
\hline & 1.0588 & \multicolumn{7}{|c|}{ !Undercoordination parameter } \\
\hline & 4.6000 & \multicolumn{7}{|c|}{ !Triple bond stabilisation parameter } \\
\hline & 2.1176 & \multicolumn{7}{|c|}{ !Undercoordination parameter } \\
\hline & 3.3056 & \multicolumn{7}{|c|}{ !Undercoordination parameter } \\
\hline & 38.4950 & \multicolumn{7}{|c|}{ !Triple bond stabilization energy } \\
\hline & 0.0000 & \multicolumn{7}{|c|}{ ! Lower Taper-radius } \\
\hline & 10.0000 & \multicolumn{7}{|c|}{ !Upper Taper-radius } \\
\hline & 2.8793 & \multicolumn{7}{|l|}{ ! Not used } \\
\hline & 33.8667 & !Valency un & dercoordi & nation & & & & \\
\hline & 6.0891 & !Valency an & gle/lone & pair para & meter & & & \\
\hline & 1.0563 & !Valency an & gle & & & & & \\
\hline & 2.0384 & !Valency an & gle paran & eter & & & & \\
\hline & 6.1431 & ! Not used & & & & & & \\
\hline & 6.9290 & !Double bon & $\mathrm{d} / \mathrm{angle} \mathrm{p}$ & arameter & & & & \\
\hline & 0.3989 & !Double bon & $\mathrm{d}$ /angle $\mathrm{r}$ & arameter: & overcool & & & \\
\hline & 3.9954 & !Double bon & $\mathrm{d}$ /angle $\mathrm{p}$ & arameter: & overcool & & & \\
\hline & -2.4837 & ! Not used & & & & & & \\
\hline & 5.7796 & !Torsion/BO & paramete & & & & & \\
\hline & 0.0000 & !Torsion ov & ercoordir & ation & & & & \\
\hline & 1.9487 & !Torsion ov & ercoordir & ation & & & & \\
\hline & -1.2327 & !Conjugatio & n 0 (not & used) & & & & \\
\hline & 2.1645 & !Conjugatio & & & & & & \\
\hline & 1.5591 & !vdWaals sh & ielding & & & & & \\
\hline & 0.1000 & !Cutoff for & bond orc & er $(* 100)$ & & & & \\
\hline & 1.7602 & !Valency an & gle conju & gation pa & rameter & & & \\
\hline & 0.6991 & ! Overcoordi & nation pa & rameter & & & & \\
\hline & 50.0000 & ! Overcoordi & nation pa & rameter & & & & \\
\hline & 1.8512 & !Valency/lo & ne pair $\mathrm{p}$ & arameter & & & & \\
\hline & 0.5000 & ! Not used & & & & & & \\
\hline & 0.0000 & ! Not used & & & & & & \\
\hline & 5.0000 & !Molecular & energy ( $r$ & ot used) & & & & \\
\hline & 0.0000 & !Molecular & energy ( $r$ & ot used) & & & & \\
\hline & 0.7903 & !Valency an & gle conju & gation pa & rameter & & & \\
\hline 6 & ! Nr & $\begin{array}{l}\text { of atoms; } \\
\text { alfa;gamma } \\
\text { cov r3; Elp } \\
\text { ov/un; vall }\end{array}$ & $\begin{array}{l}\text { cov.r; va } \\
\text { vdW; valer } \\
\text {; Heat inc } \\
\text {; n.u.; ival }\end{array}$ & $\begin{array}{l}\text { lency;a.m } \\
\text { cy; Eunder } \\
. ; \text { n.u.; n. } \\
\text { 3, vval4 }\end{array}$ & $\begin{array}{l}\text {; Rvdw; Evc } \\
; \text { Eover; ch } \\
\text { u.; n.u. ;r }\end{array}$ & $\begin{array}{l}\text {; gammaEE } \\
\text { EMM; etaE } \\
\text { a. }\end{array}$ & $\begin{array}{l}\text { 1; cov.r2 } \\
\text { im; n.u. }\end{array}$ & \\
\hline $\mathrm{C}$ & 1.367 & 4.0000 & 12.0000 & 2.0453 & 0.1444 & 0.8485 & 1.1706 & 4.0000 \\
\hline & 9.000 & 1.5000 & 4.0000 & 30.0000 & 79.5548 & 4.8446 & 7.0000 & 0.0000 \\
\hline & 1.116 & 0.0000 & 181.0000 & 14.2732 & 24.4406 & 6.7313 & 0.8563 & 0.0000 \\
\hline & -4.1021 & 5.0000 & 1.0564 & 4.0000 & 2.9663 & 0.5000 & 0.1000 & 12.0000 \\
\hline $\mathrm{H}$ & 0.893 & 1.0000 & 1.0080 & 1.3550 & 0.0930 & 0.8203 & -0.1000 & 1.0000 \\
\hline & 8.223 & 33.2894 & 1.0000 & 0.0000 & 121.1250 & 3.7248 & 9.6093 & 1.0000 \\
\hline & -0.100 & 0.0000 & 55.1878 & 3.0408 & 2.4197 & 0.0003 & 1.0698 & 0.0000 \\
\hline & -19.457 & 4.2733 & 1.0338 & 1.0000 & 2.8793 & 0.5000 & 0.1000 & 12.0000 \\
\hline Ar & -0.100 & 2.0000 & 39.9480 & 1.9200 & 0.3200 & 0.5000 & -0.1000 & 4.0000 \\
\hline & 12.000 & 4.0000 & 4.0000 & 0.0000 & 0.0000 & 5.0000 & 13.0000 & 0.0000 \\
\hline & -0.100 & 0.0000 & -2.3700 & 6.4918 & 8.5961 & 0.2368 & 0.8563 & 0.0000 \\
\hline & -5.000 & 3.1873 & 1.0338 & 6.2998 & 2.5791 & 2.5849 & 8.8277 & 14.6113 \\
\hline $\mathrm{He}$ & -0.100 & 2.0000 & 4.0026 & 1.3000 & 0.0050 & 0.5000 & -0.1000 & 4.0000 \\
\hline & 12.000 & 4.0000 & 4.0000 & 0.0000 & 0.0000 & 5.0000 & 13.0000 & 0.0000 \\
\hline
\end{tabular}




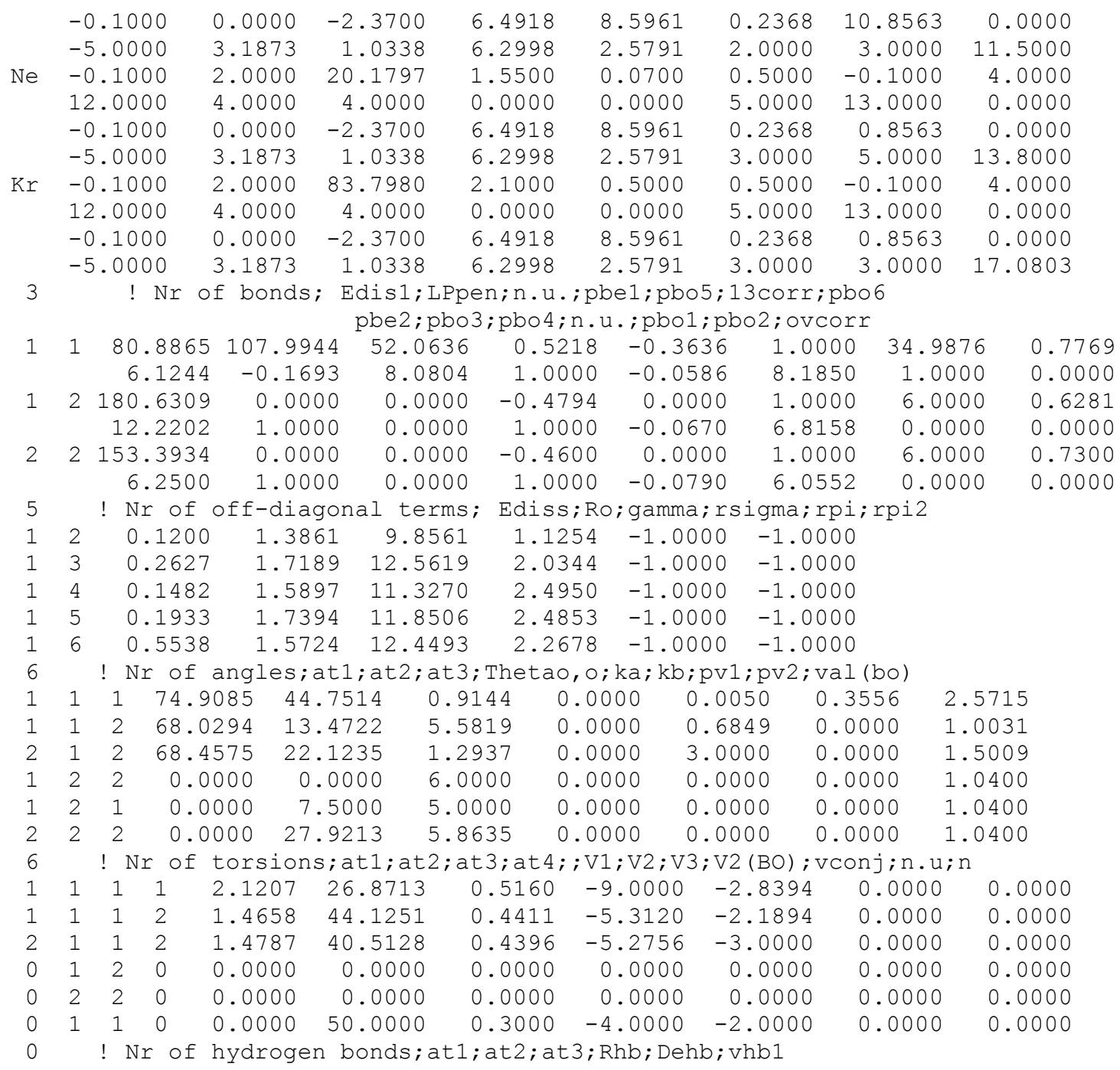




\section{S3. Simulation model}

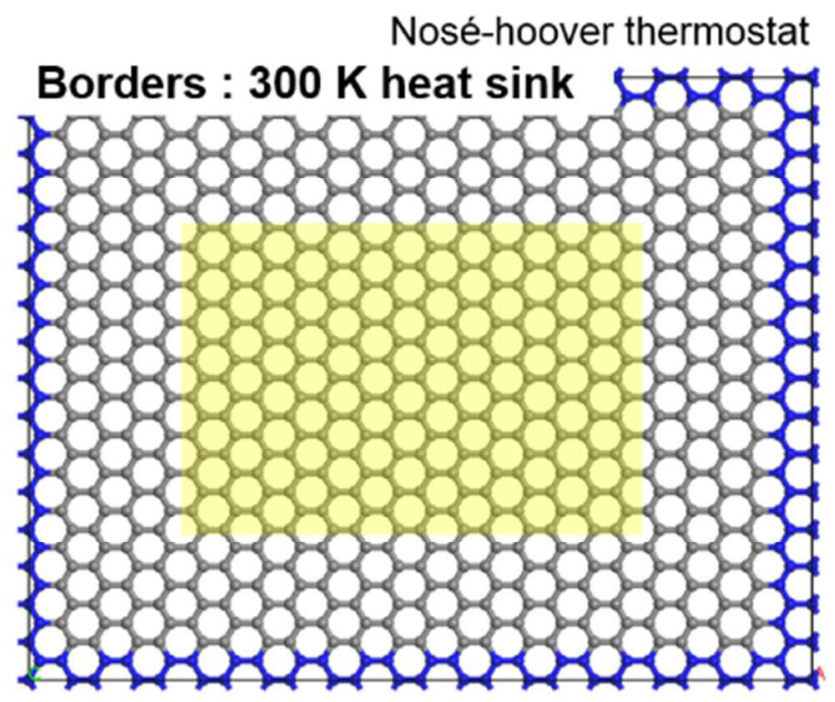

Figure S2. Simulation model: the periodic graphene sheet with approximate dimension of $52 \times 40$ $\AA^{2}$. Ions were irradiated within the center area $\left(30 \times 20 \AA^{2}\right)$ of graphene, indicated as the yellow square. The periodic edges were kept at $300 \mathrm{~K}$ (by Nosé-hover thermostat), playing the role of heat sink during MD simulations. 


\section{S4. Irradiated graphene with coordinatively unsaturated atoms colored by blue}

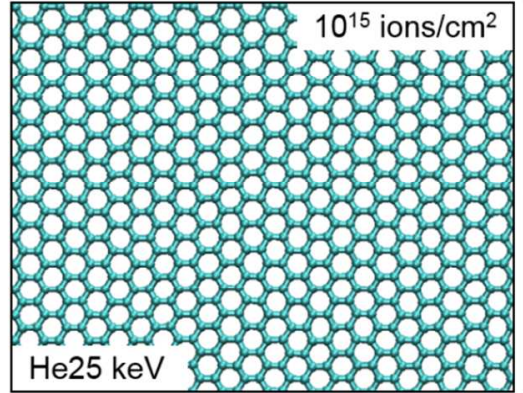

Annealing \&

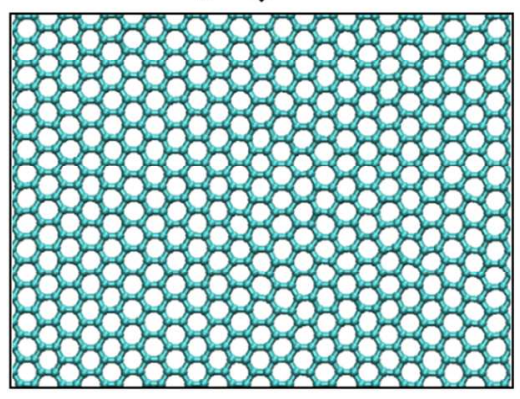

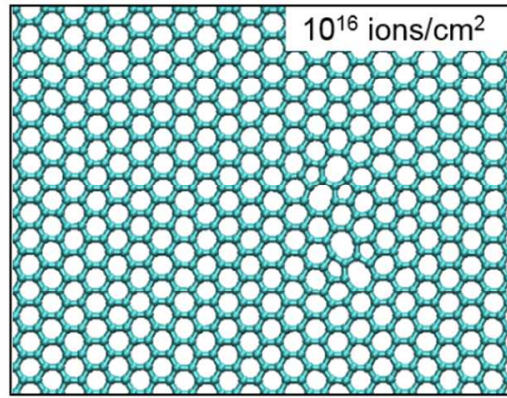

Љ

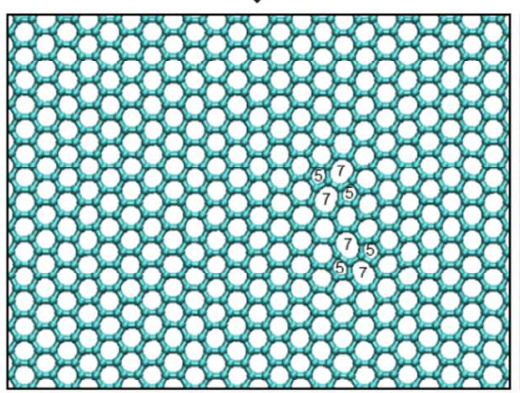

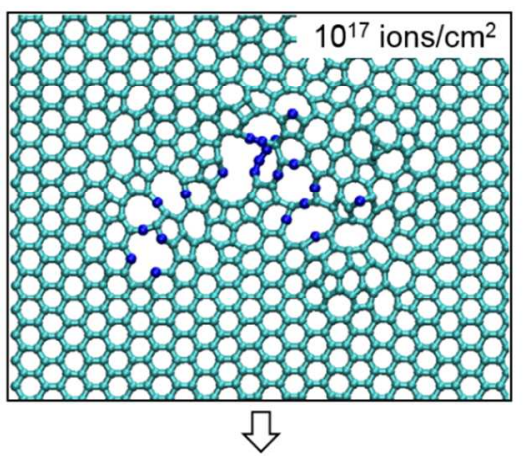
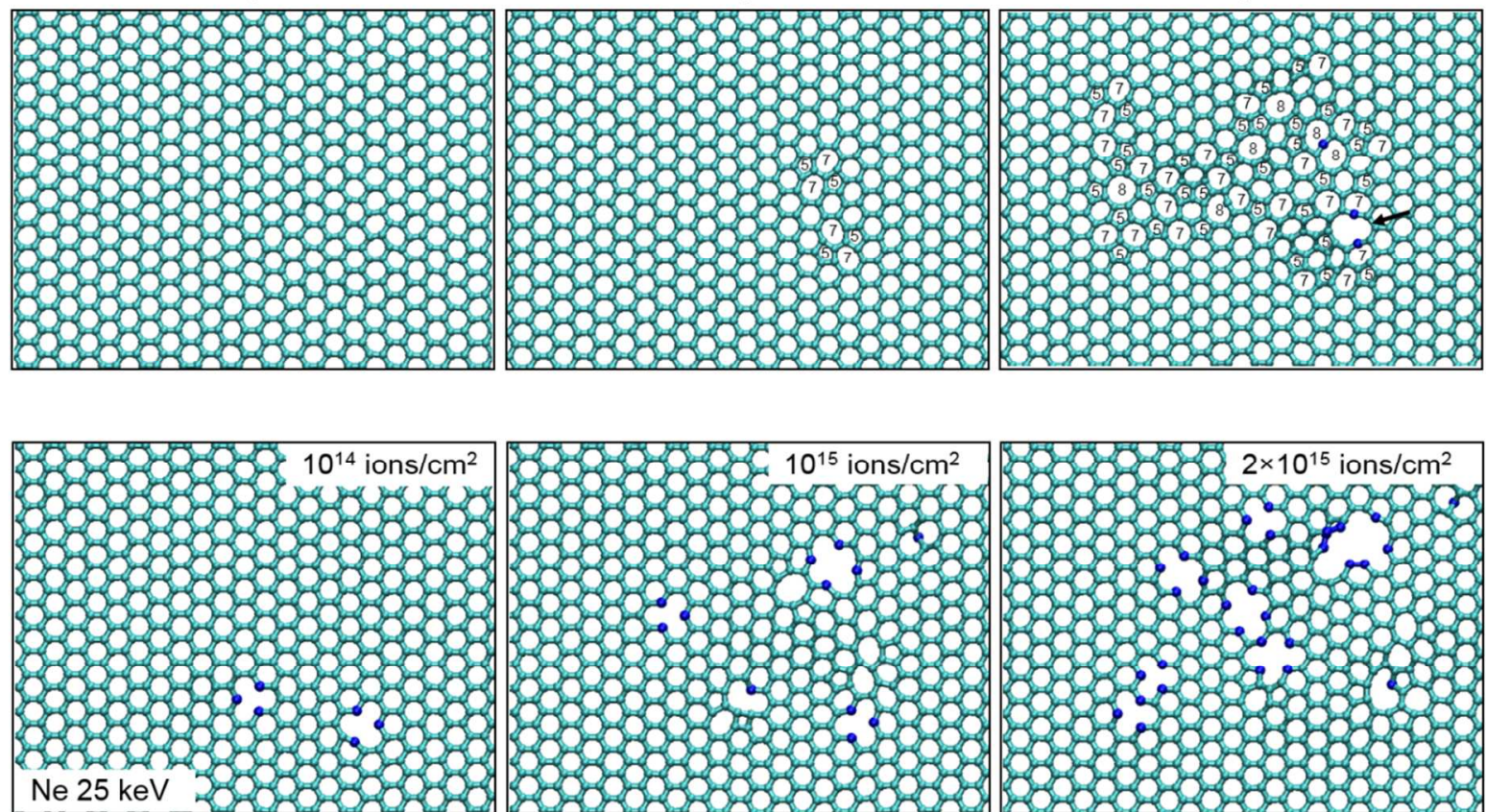

Annealing $\&$
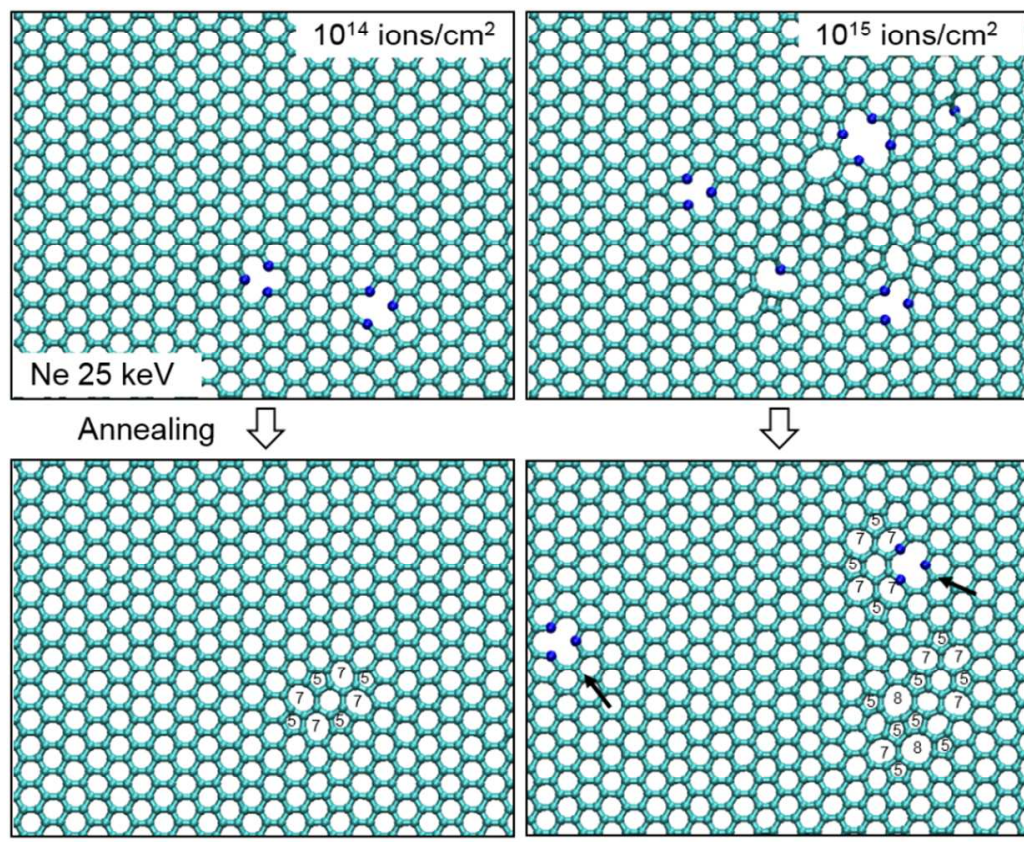

凸
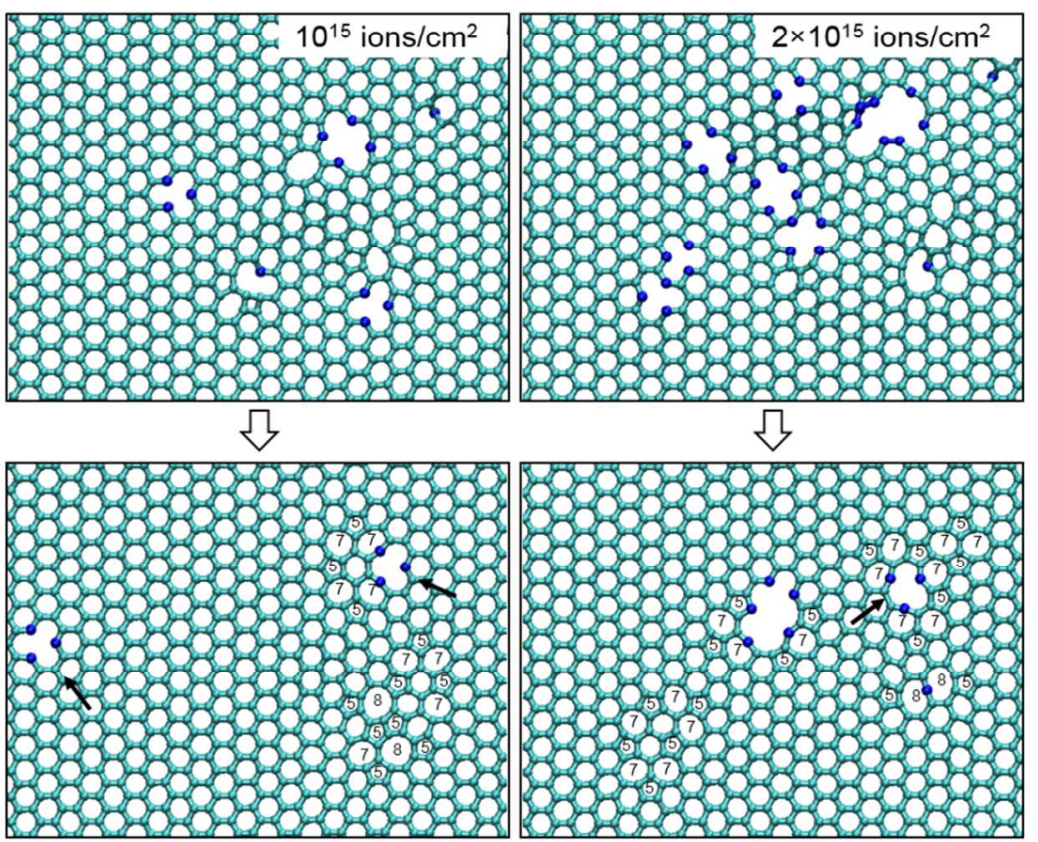

Figure S3. $\mathrm{He}^{+}$and $\mathrm{Ne}^{+}$Irradiated graphene with coordinatively unsaturated atoms colored by

blue. (Original figures are from Fig. 1 and Fig. 2 in the manuscript). 


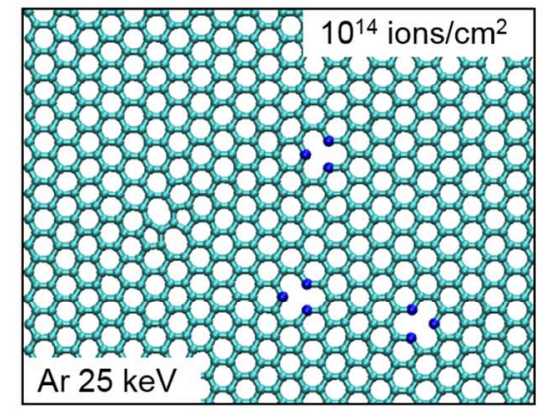

Annealing \&

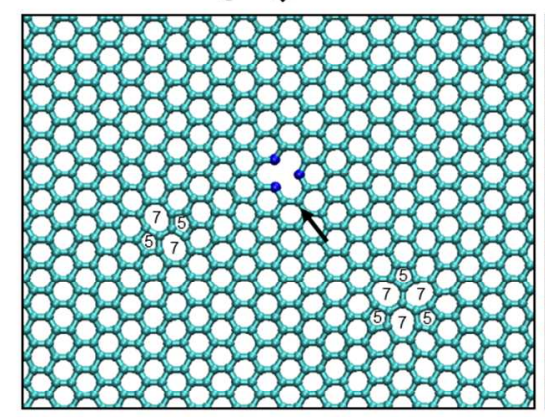

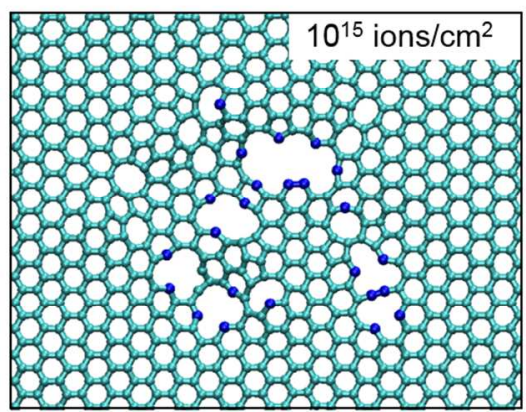

$\checkmark$

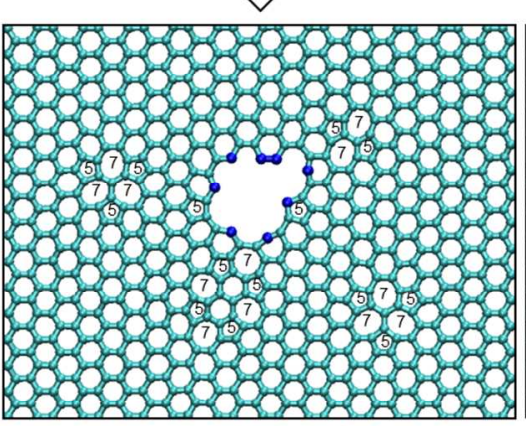

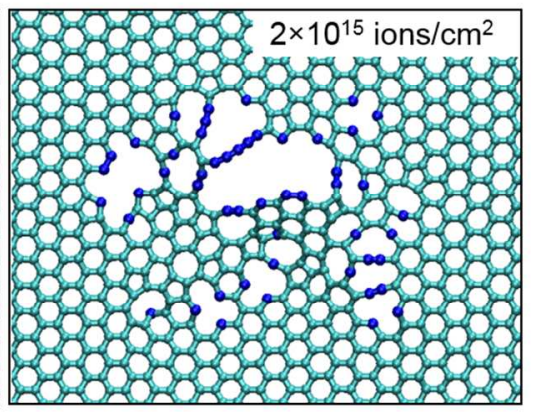

$\checkmark$

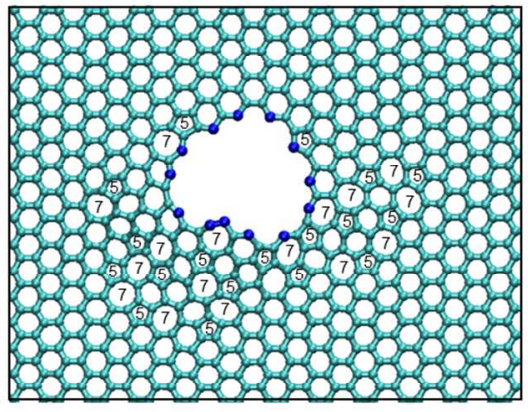

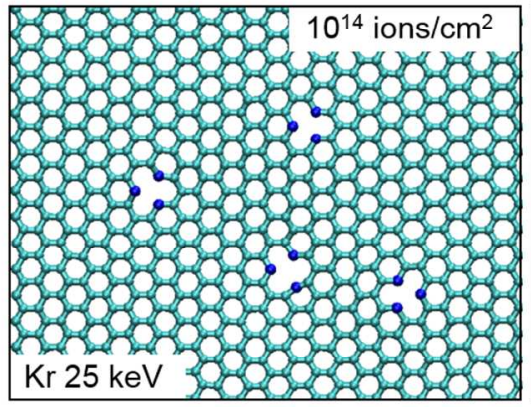

Annealing \&

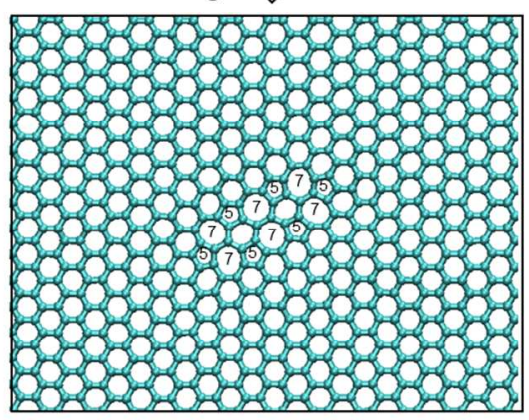

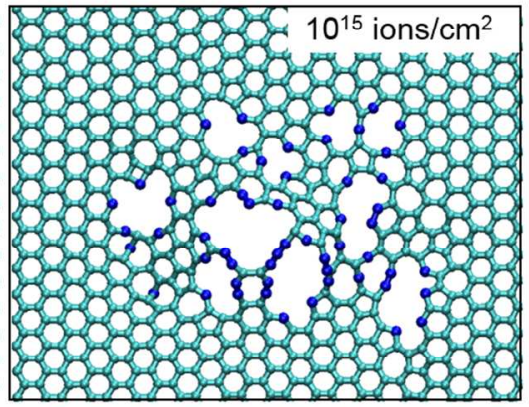

\3.

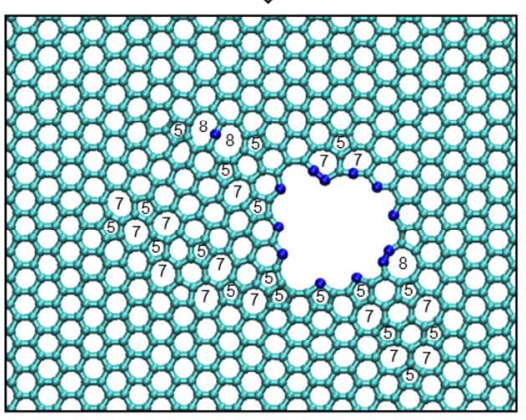

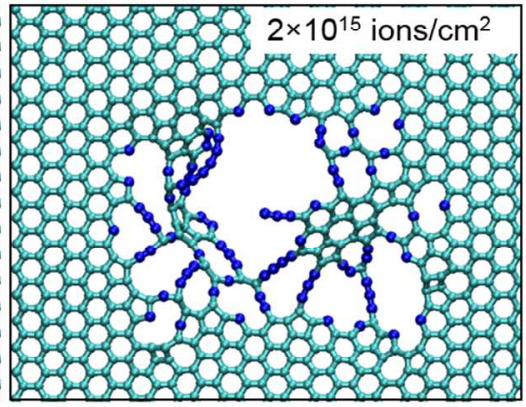

ऽ

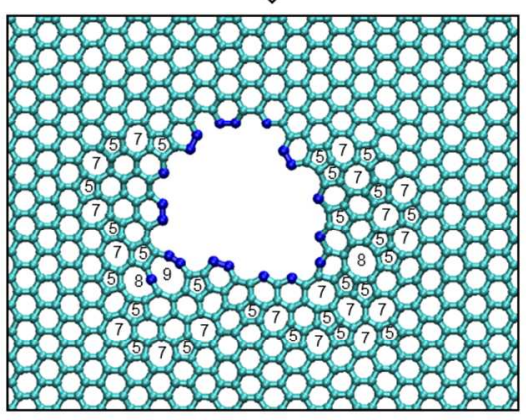

Figure S4. $\mathrm{Ar}^{+}$and $\mathrm{Kr}^{+}$Irradiated graphene with coordinatively unsaturated atoms colored by

blue. (Original figure is from Fig. 3 in the manuscript). 

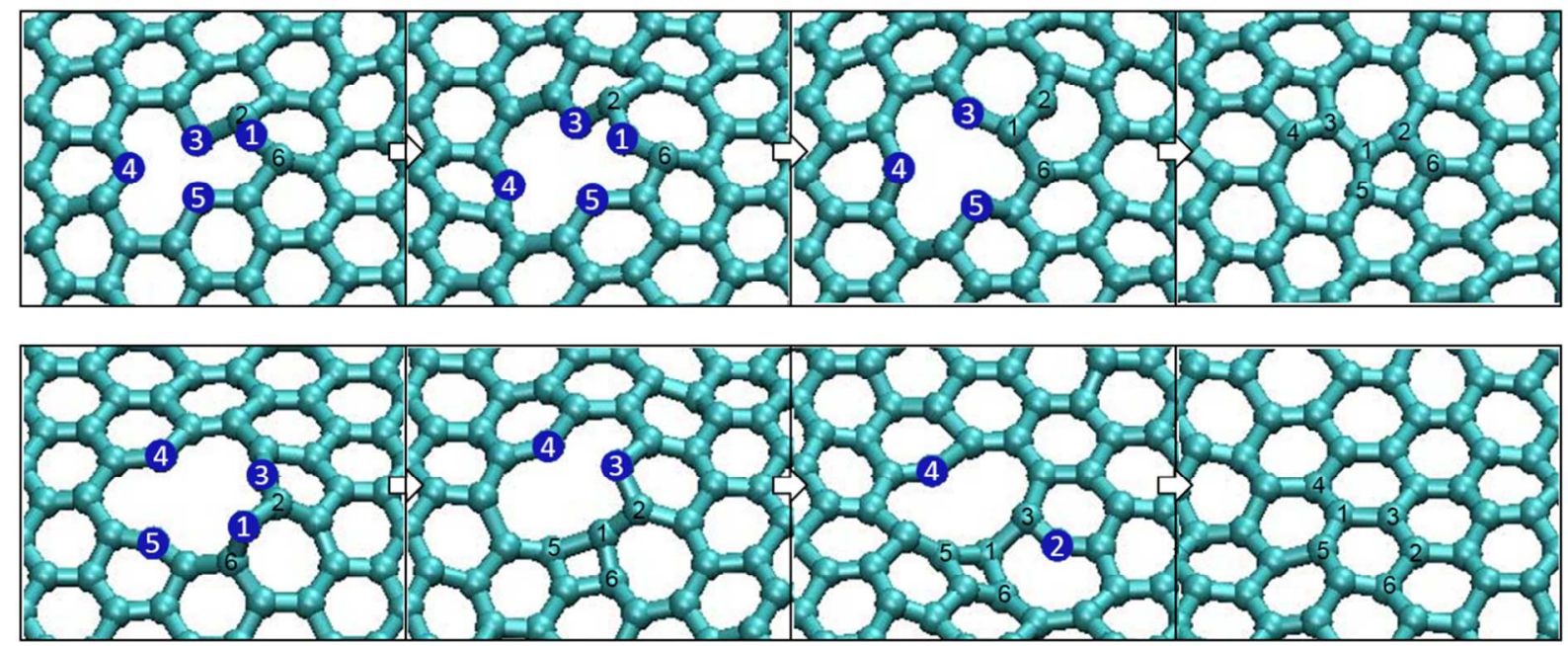

Figure S5. Dynamic processes of the representative reconstruction of Frenkel defects. Coordinatively unsaturated atoms were colored by blue. (Original figure is from Fig. 6 in the manuscript). 

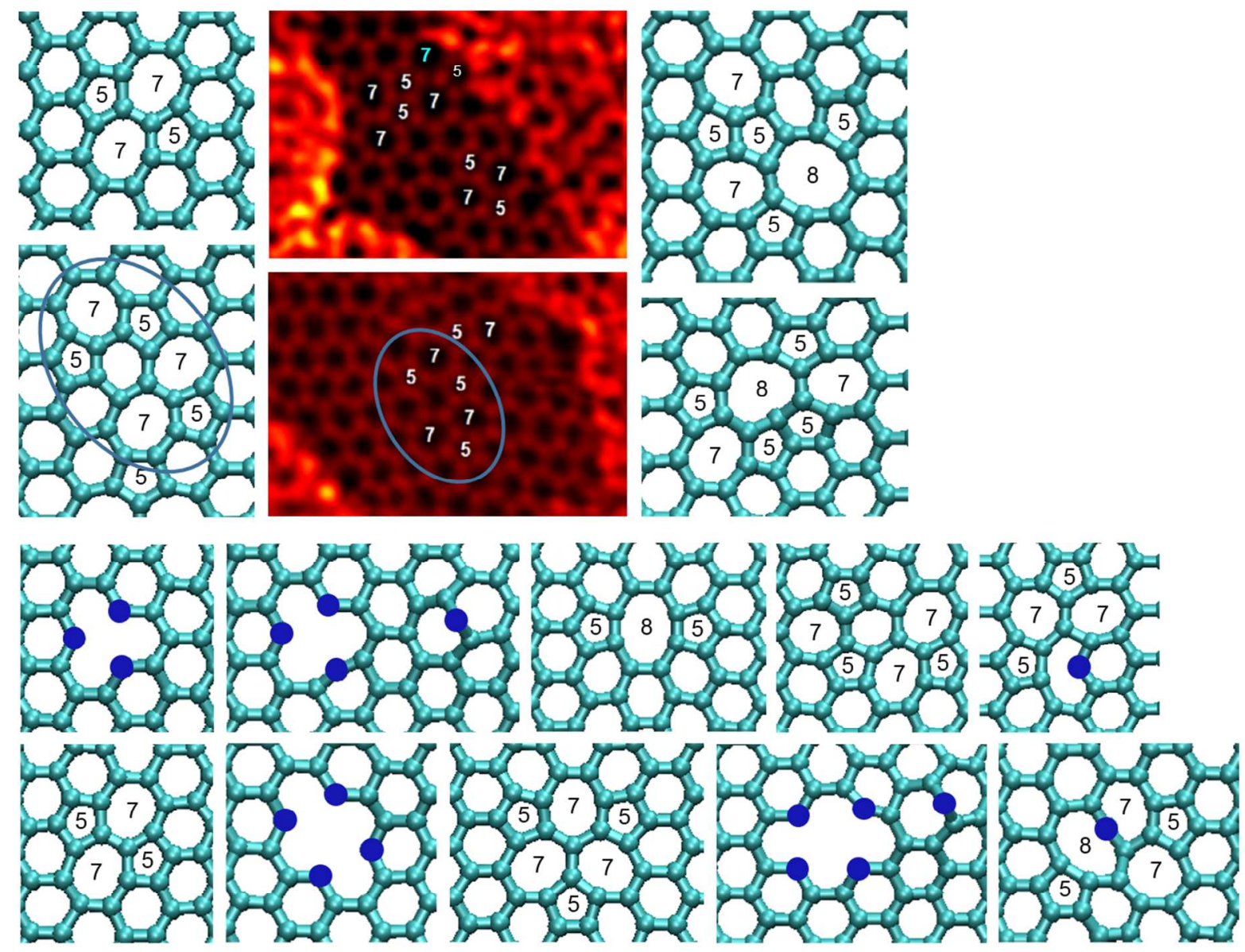

Figure S6. The types of defects that were observed more than three times in 100 simulations of ion irradiation. Coordinatively unsaturated atoms were colored by blue. (Original figure is from Fig. 7 in the manuscript). 


\section{S5. Quantitative Image Analysis of Graphene Defects from STEM Images}

To quantitatively measure the defect configurations that were induced as a result of $\mathrm{He}^{+}$ and $\mathrm{Ne}^{+}$irradiation, atomic resolution STEM imaging was performed. Figs. S7 (a) and S8 (a) display the aberration-corrected STEM images that were presented in Figs 1 and 2 in the main manuscript. Atom finding and defect configurations are presented in Figs S7 (b) and S8 (b), which correspond to the experimental STEM images processed to find the local maxima using a 2D Gaussian blur function. The $\mathrm{C}$ rings were automatically identified in the regions not covered by hydrocarbon contamination and the number of edges was calculated for each ring. For $\mathrm{He}^{+}$ irradiation at $10^{15}, 10^{16}$, and $10^{17}$ ions $/ \mathrm{cm}^{2}$ there were 942,102 , and 123 rings, respectively. For $\mathrm{Ne}^{+}$irradiation at $1.5 \times 10^{14}, 6.22 \times 10^{14}$, and $2.55 \times 10^{15} \mathrm{ions} / \mathrm{cm}^{2}, 184,172$, and 159 rings were found. The full images with $\mathrm{C}-\mathrm{C}$ bond plot and edge number are shown in the Figs. S7 (b) and S8 (b). The statistics of rings with different number of edges were plotted Fig S9. For both $\mathrm{He}^{+}$ and $\mathrm{Ne}^{+}$irradiation, we can see as the irradiation dose increases, the number of 6-member rings decreases. 
a)

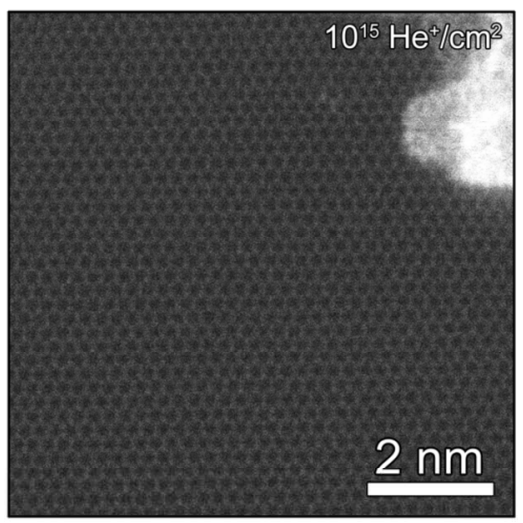

b)

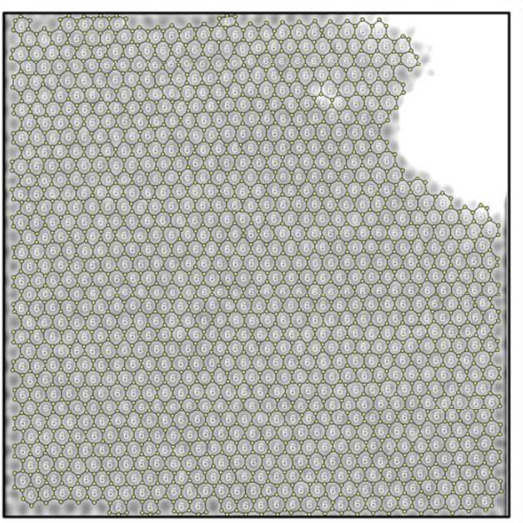

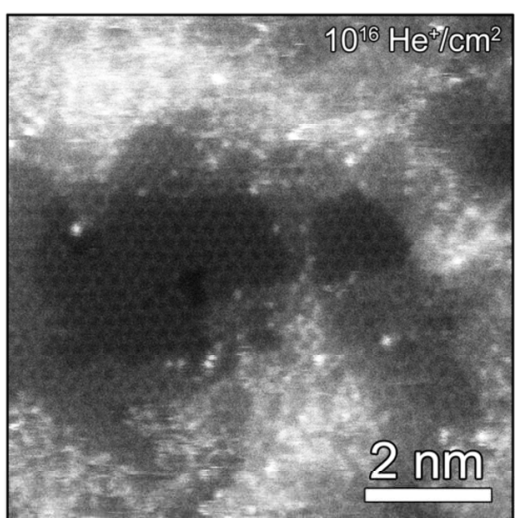
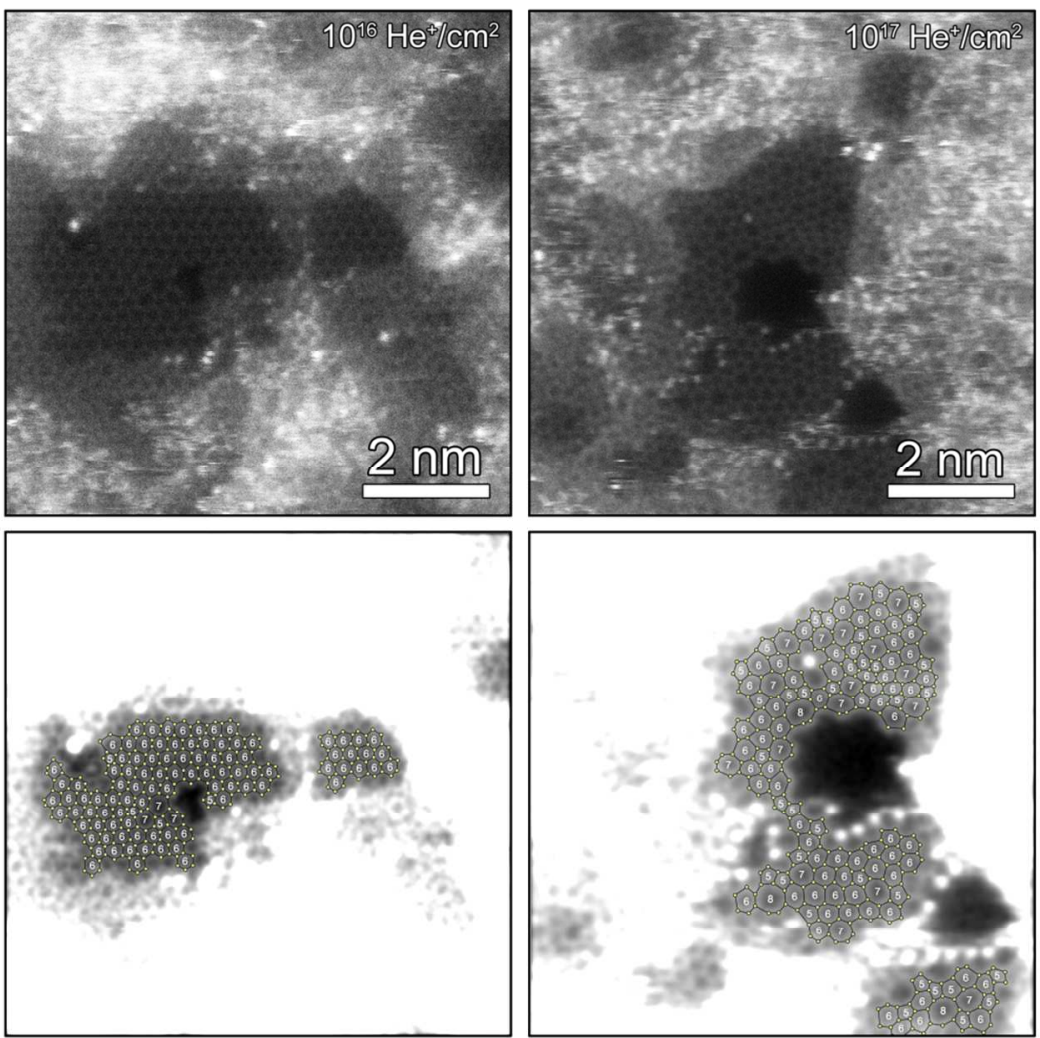

Figure S7. (a) Aberration-corrected STEM images of $\mathrm{He}^{+}$ion irradiated graphene at $10^{15}, 10^{16}$, and $10^{17}$ ions $/ \mathrm{cm}^{2}$. (b) Corresponding quantitative image analysis performed using a $2 \mathrm{D}$ Gaussian blur to determine the atom location and graphene defect configuration for atomically resolved areas. Bright areas of heavy hydrocarbon surface contamination were not analyzed. 
a)

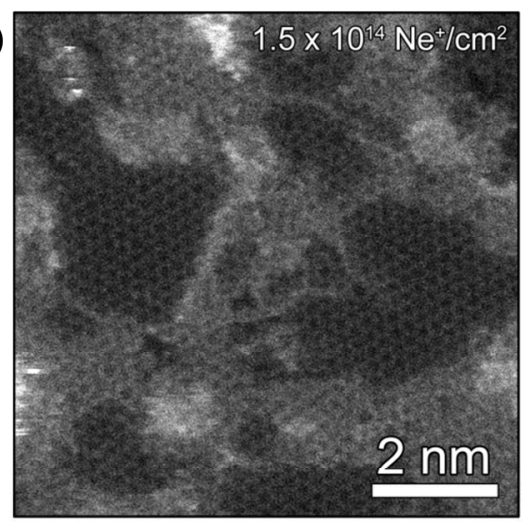

b)

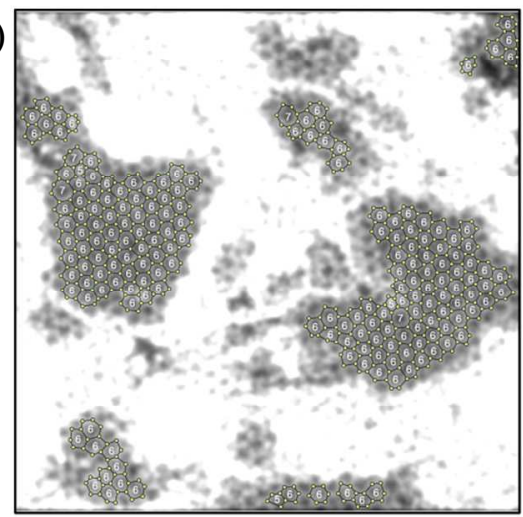

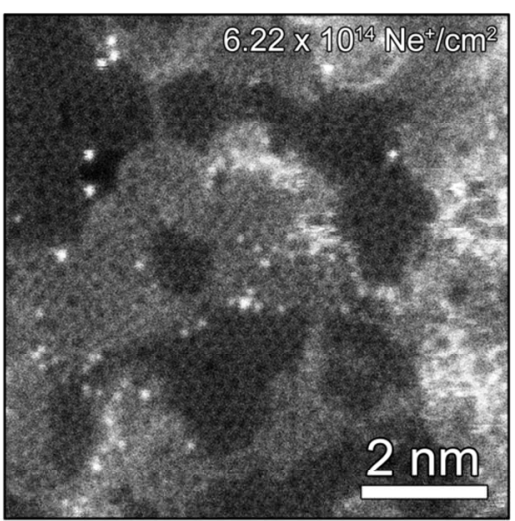
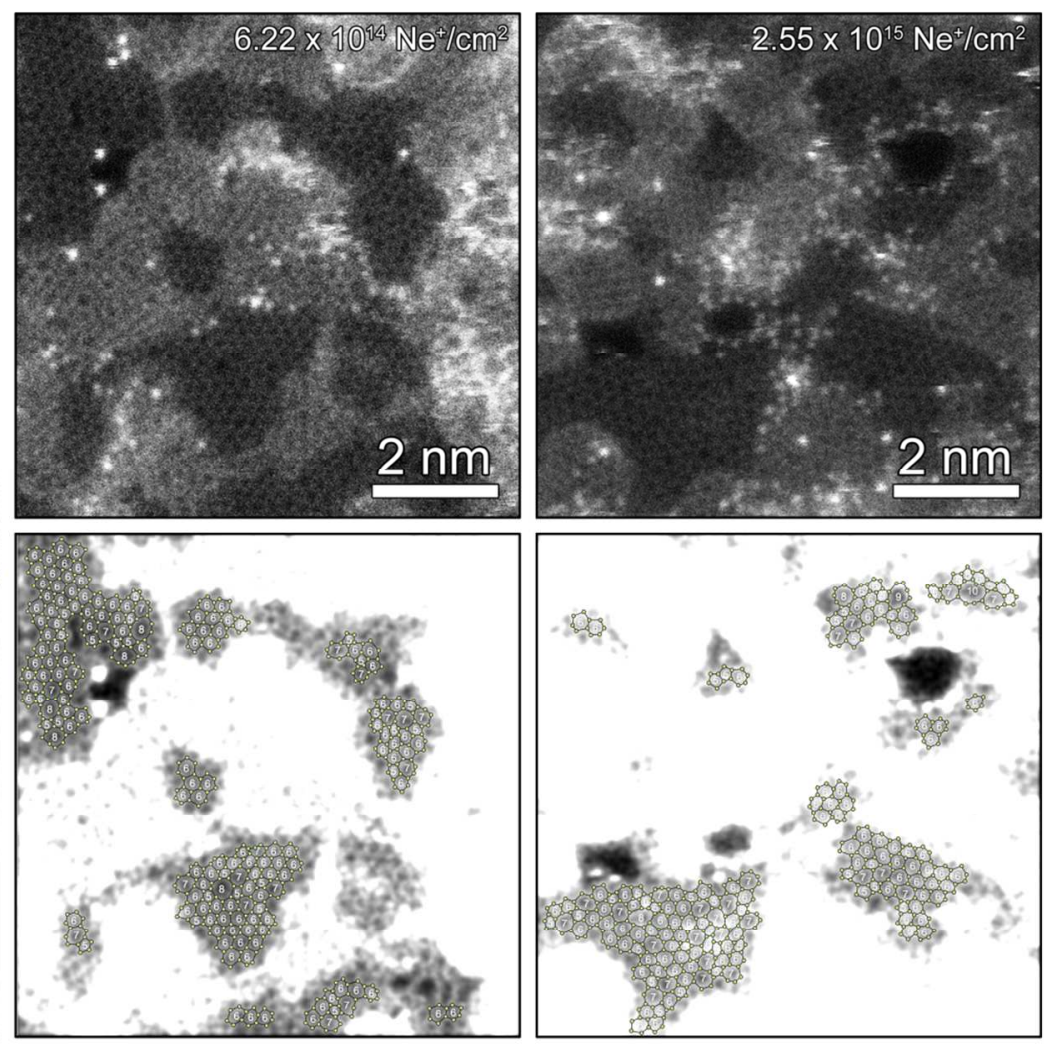

Figure S8. (a) Aberration-corrected STEM images of the $\mathrm{Ne}^{+}$irradiated graphene at $1.5 \times 10^{14}$, $6.22 \times 10^{14}$, and $2.55 \times 10^{15} \mathrm{ions} / \mathrm{cm}^{2}$. (b) Corresponding quantitative image analysis performed using a 2D Gaussian blur to determine the atom location and graphene defect configuration for atomically resolved areas. Bright areas of heavy hydrocarbon surface contamination were not analyzed. 

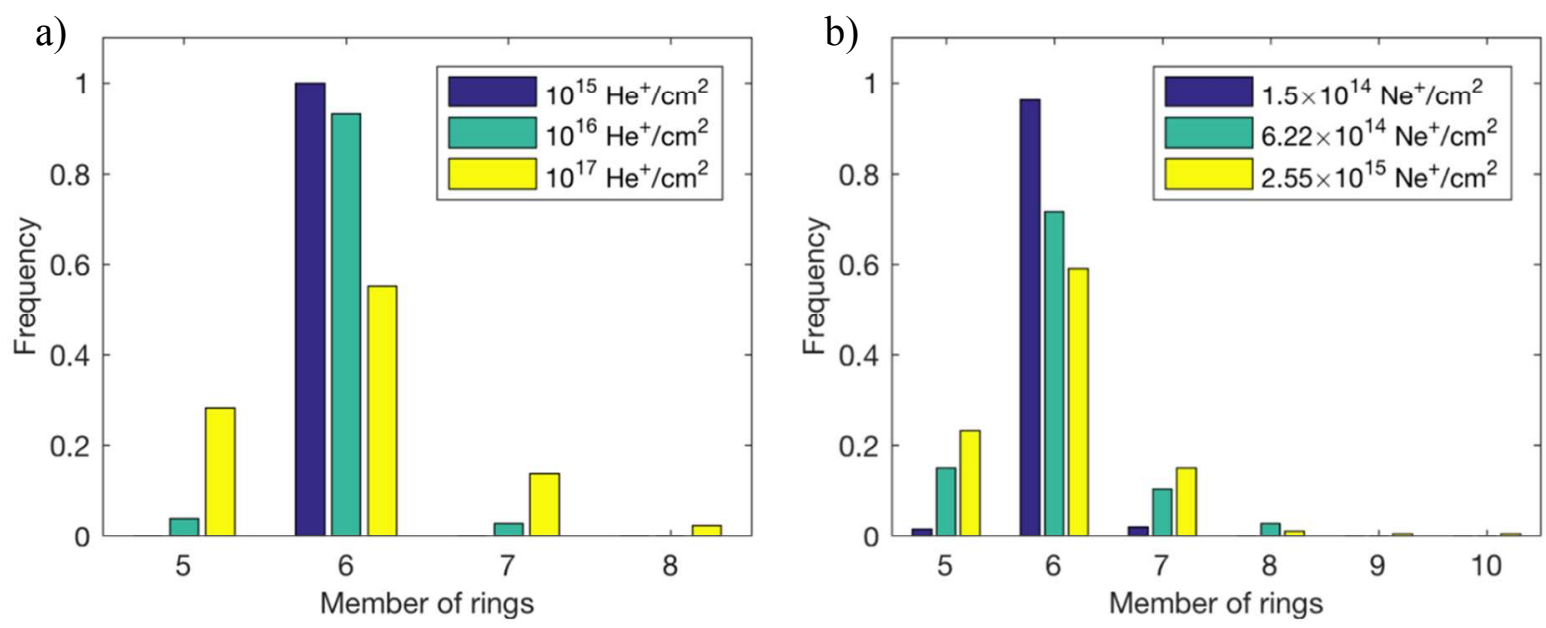

Figure S9. Statistical analysis from experimental STEM image analysis of graphene defects induced by (a) $\mathrm{He}^{+}$ion irradiation and (b) $\mathrm{Ne}^{+}$ion irradiation. 


\section{S6. Effects of relaxation before additional ion impacts on defect density}

Due to computational limit, it is almost impossible to allow for a full relaxation of graphene between every individual impact. In order to address the effects of relaxation before additional ion impacts, we have run several test simulations, in which graphene layers that were initially irradiated by $\mathrm{He}^{+} 10^{16}$ ions $/ \mathrm{cm}^{2}, \mathrm{Ne}^{+} 10^{15}$ ions $/ \mathrm{cm}^{2}, \mathrm{Ar}^{+} 10^{15}$ ions $/ \mathrm{cm}^{2}$, and $\mathrm{Kr}^{+} 10^{15}$ ions $/ \mathrm{cm}^{2}$ and that were subsequently annealed at high temperature, were further irradiated and annealed. In each ion irradiation, two independent simulations were performed. The displaced carbon atoms (\%) and crystallinities (\%) were obtained and indicated as X in Fig. S10 with colors corresponding to each ion. All the data were located within the error ranges or very close to the error limits, indicating that the effects of relaxation before the additional ion impacts do not seem to be significantly affecting the defect density.
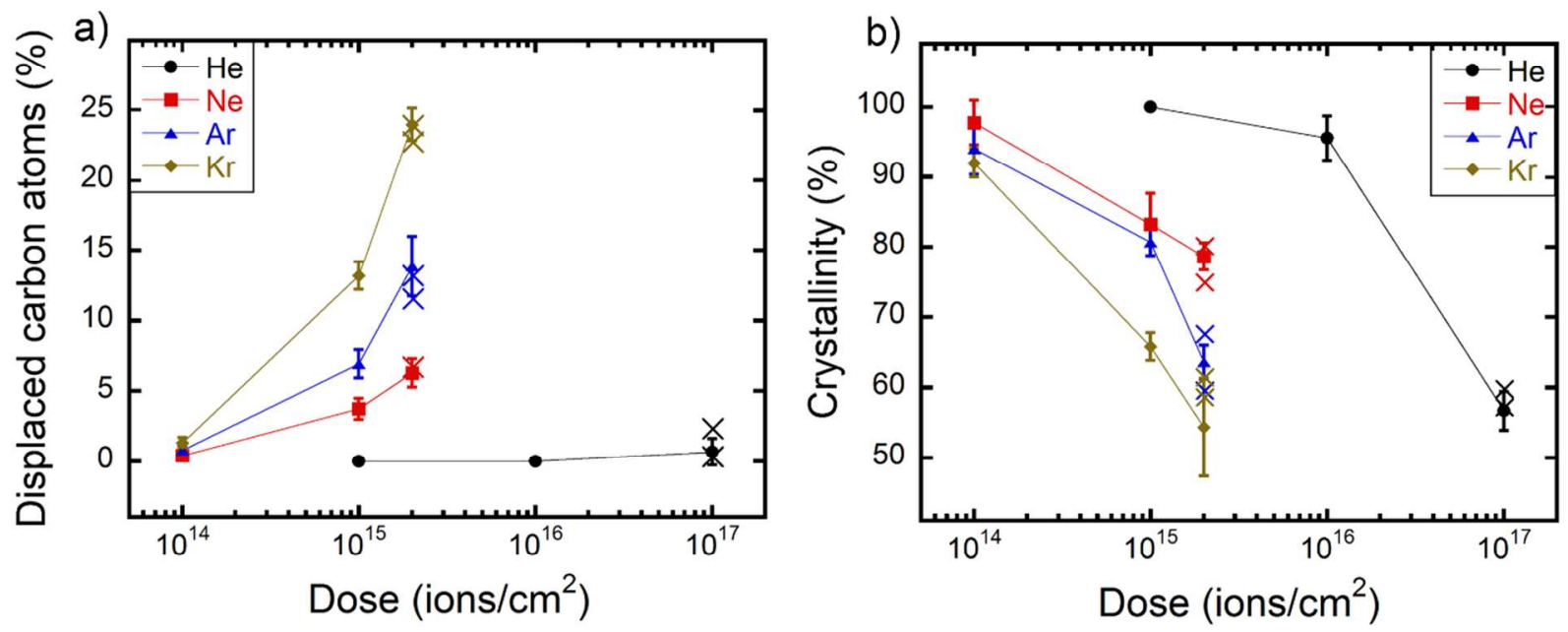

Figure S10. Quantitative measures of the defect density in the irradiated graphene. Data points indicated as $\mathrm{X}$ with colors corresponding to each ion were obtained from the irradiation and subsequent annealing of graphene layers that were initially irradiated by $\mathrm{He}^{+} 10^{16}$ ions $/ \mathrm{cm}^{2}, \mathrm{Ne}^{+}$ $10^{15}$ ions $/ \mathrm{cm}^{2}, \mathrm{Ar}^{+} 10^{15}$ ions $/ \mathrm{cm}^{2}$, and $\mathrm{Kr}^{+} 10^{15}$ ions $/ \mathrm{cm}^{2}$ and subsequently annealed at high 
temperature. (Data points indicated as $\mathrm{X}$ were obtained from two independent simulations in each ion irradiation, and were added to the original Figure 4 in the manuscript). 


\section{S7. Functionalization of nanopore edges}

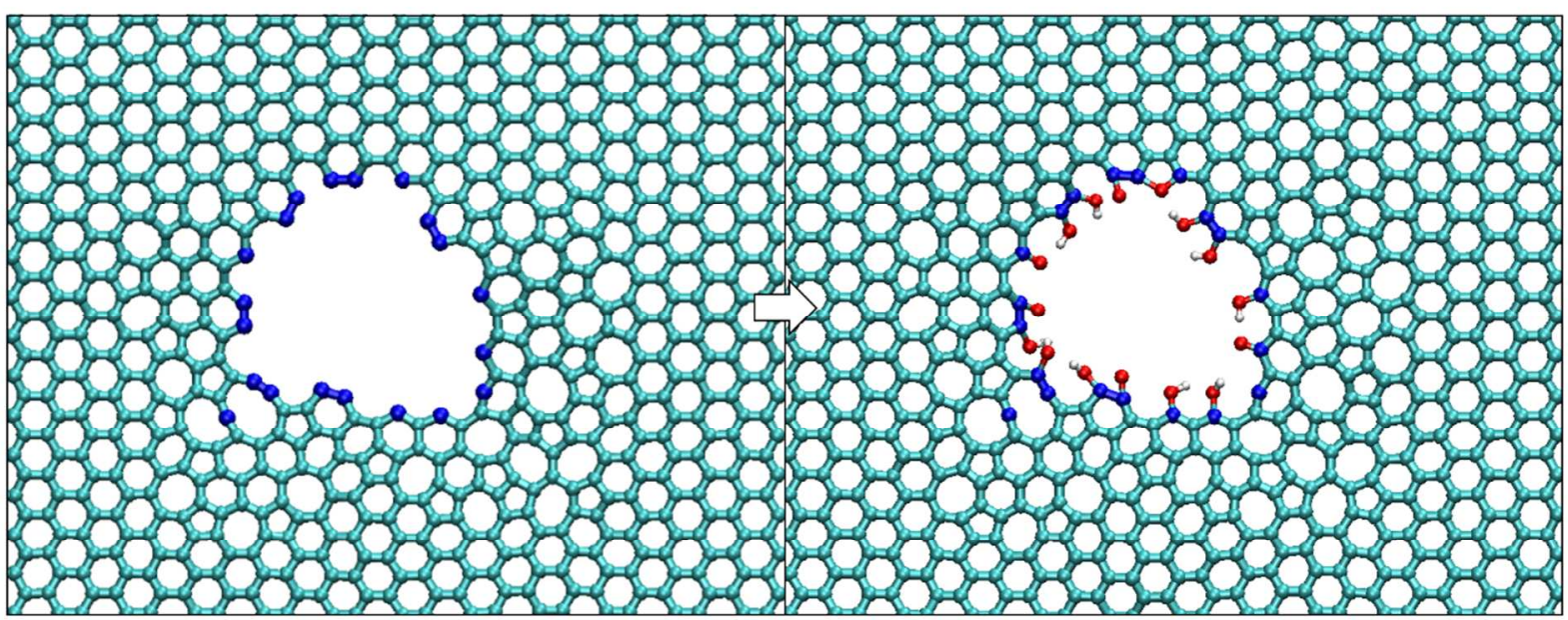

Figure S11. Nanopore edges functionalized either by hydroxyl groups or atomic oxygen forming epoxide-like arrangement in the hybrid grand canonical Monte Carlo/molecular dynamics (GC$\mathrm{MC} / \mathrm{MD}$ ) simulations. Initial geometry was taken after $\mathrm{Kr}^{+} 2 \times 10^{15}$ ions $/ \mathrm{cm}^{2}$ irradiation, followed by high temperature annealing. (cyan atoms: coordinatively saturated carbon atoms in graphene; blue atoms: coordinatively unsaturated carbon atoms in graphene; red atoms: oxygen atoms; white atoms: hydrogen atoms) 


\section{REFERENCES}

1. Ziegler, J. F.; Biersack, J. P.; Littmark, U., The Stopping and Range of Ions in Solids. Pergamon: New York, 1985. 